Developmental plasticity and transgenerational reprogramming following vitrified embryo transfer in Oryctolagus cuniculus

\title{
INDEX OF CONTENTS
}




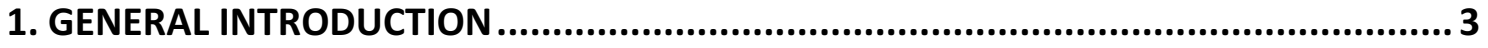

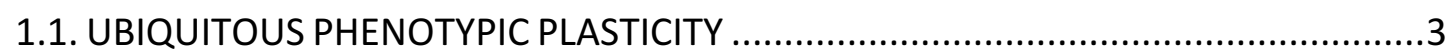

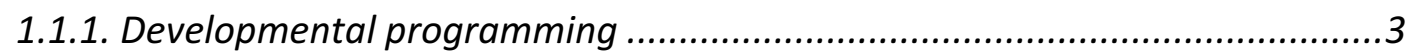

1.1.2. Transgenerational developmental programming ....................................... 3

1.1.3. Epigenetic mechanisms ....................................................................... 4

1.2. CRITICAL WINDOW OF THE PREIMPLANTATION PERIOD ...................................

1.3. CONSEQUENCES OF INTERCEDING PREIMPLANTATION DEVELOPMENT ...............5

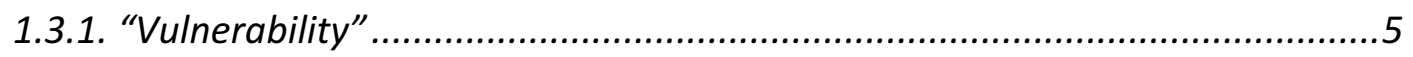

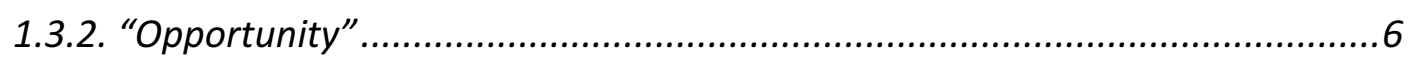

1.3.3. The advent of assisted reproduction technologies (ARTs) ...........................6

1.4. IMPLICATIONS FOR EMBRYO CRYOPRESERVATION ......................................

1.5. REFERENCES

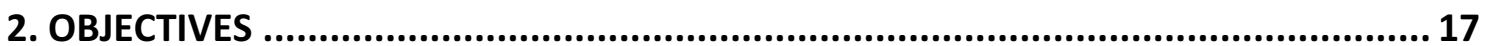

3. CHAPTER I. MINIMALLY INVASIVE EMBRYO TRANSFER AND EMBRYO VITRIFICATION AT THE OPTIMAL EMBRYO STAGE IN THE RABBIT MODEL................................... 21

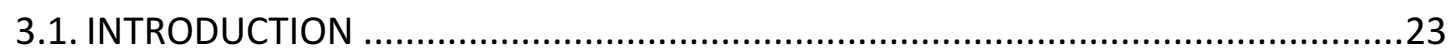

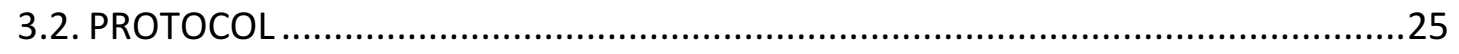

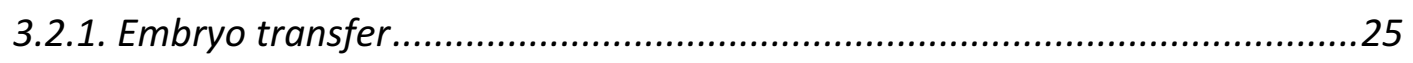

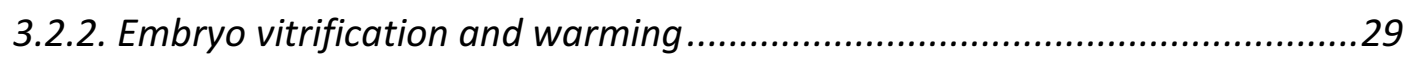

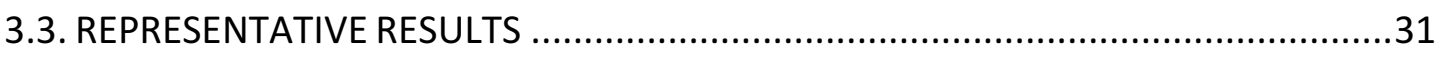

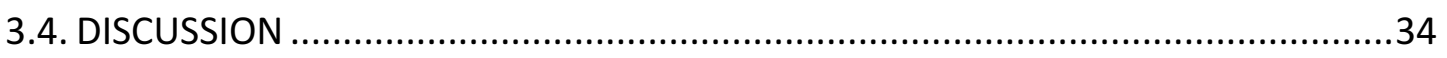

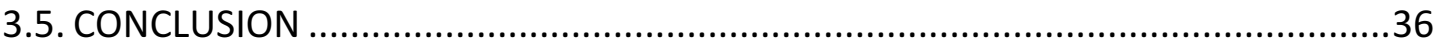

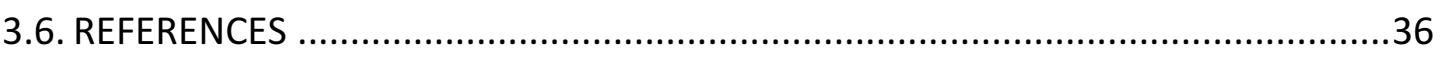

4. CHAPTER II. DEVELOPMENTAL PLASTICITY IN RESPONSE TO EMBRYO CRYOPRESERVATION: THE IMPORTANCE OF THE VITRIFICATION DEVICE IN

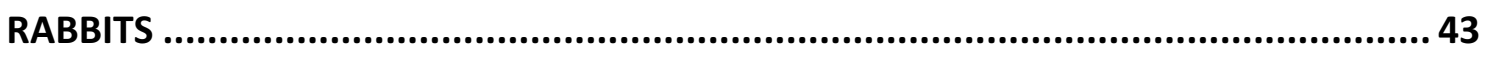

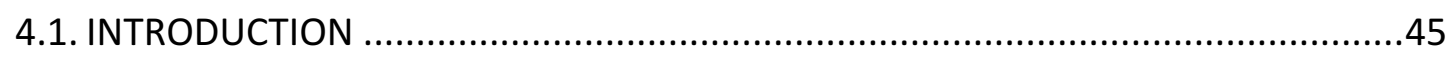

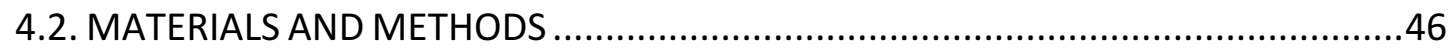

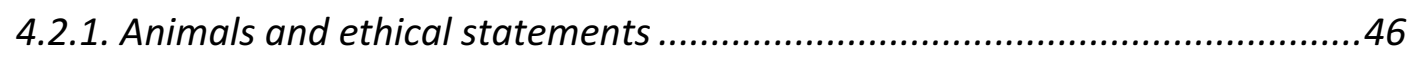

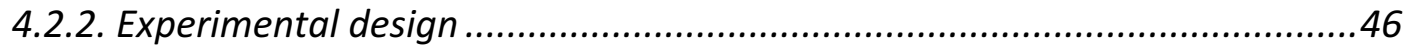

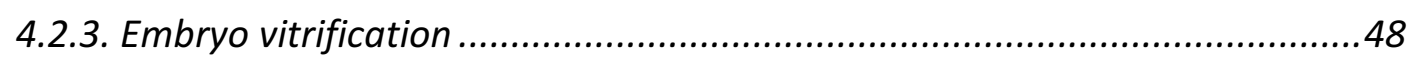

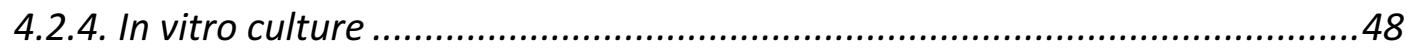

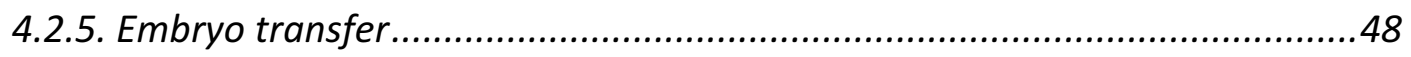

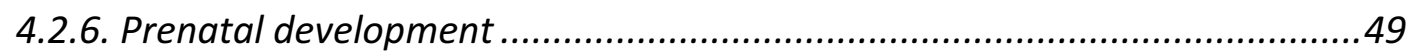

4.2.7. Postnatal growth performance and body weight study ............................49 
4.2.8. Determination peripheral blood parameters...........................................49

4.2.9. Male reproductive performance ...............................................................50

4.2.10. Female reproductive performance ........................................................51

4.2.11. Lactation performance: milk yield, milk composition and nutritional

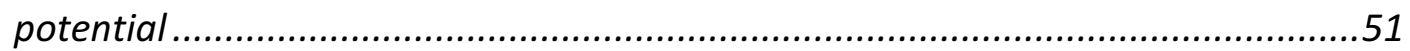

4.2.12. Statistical analysis of phenotypic data ................................................52

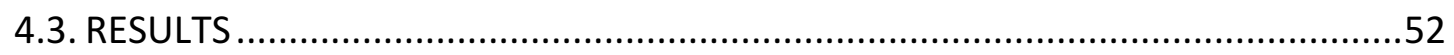

4.3.1. Effect of embryo vitrification on the embryonic in vitro development .........52

4.3.2. Prenatal survival: rates of implantation, foetal losses and offspring ...........53

4.3.3. Postnatal growth performance and body weight....................................53

4.3.4. Healthy status: Peripheral blood parameters............................................55

4.3.5. Reproductive performances....................................................................5

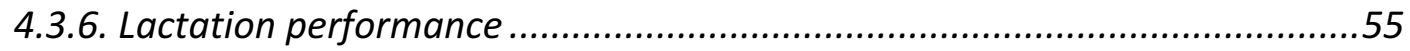

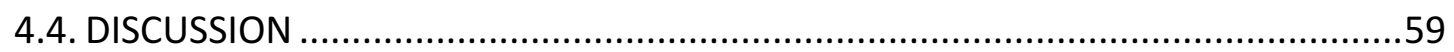

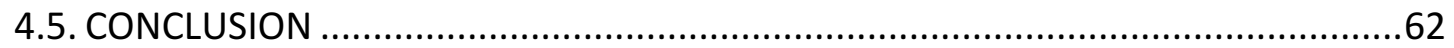

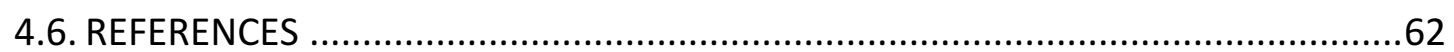

5. CHAPTER III. LONG-TERM PHENOTYPIC AND PROTEOMIC CHANGES FOLLOWING VITRIFIED EMBRYO TRANSFER IN THE RABBIT MODEL .........................................69

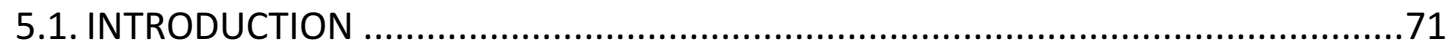

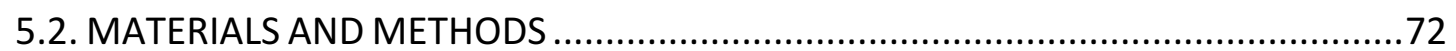

5.2.1 Animals and ethical statements ........................................................... 72

5.2.2. Vitrified embryo transfer procedure ..................................................... 73

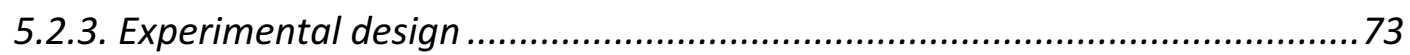

5.2.4. Growth performance during postnatal development................................ 75

5.2.5. Body weight and organ phenotypic comparison ........................................ 75

5.2.6. Determination of peripheral blood parameters ........................................75

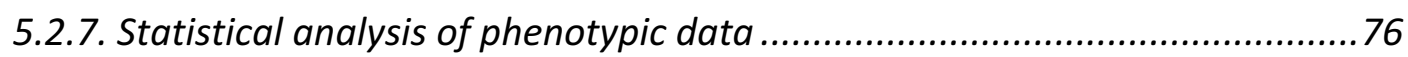

5.2.8. Comparative proteomic analysis ................................................................. 76

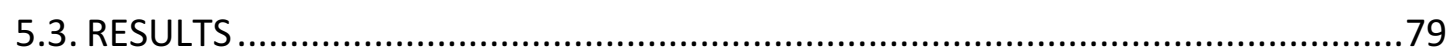

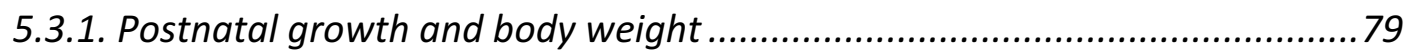

5.3.2. Body weight and organ phenotype at adulthood.....................................8 80

5.3.3. Peripheral blood parameters (healthy status)..........................................81

5.3.4. Comparative study of the liver proteome ..............................................81

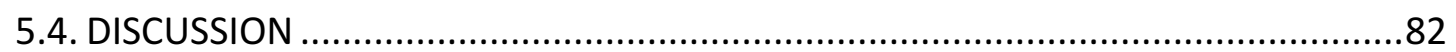

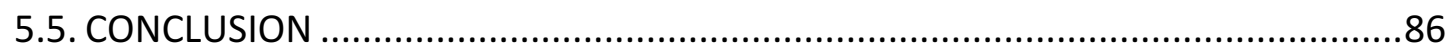

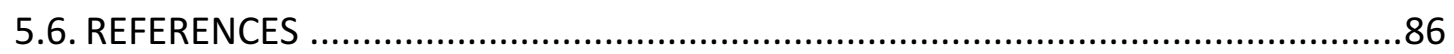


6. CHAPTER IV. LONG-TERM AND TRANSGENERATIONAL PHENOTYPIC, TRANSCRIPTIONAL AND METABOLIC EFFECTS IN RABBIT MALES BORN FOLLOWING

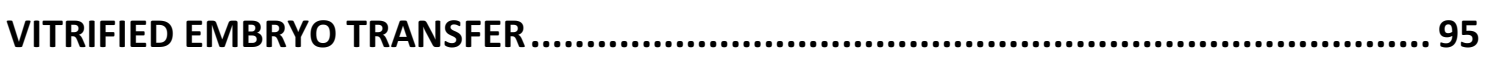

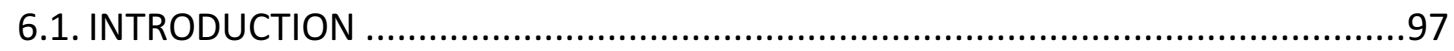

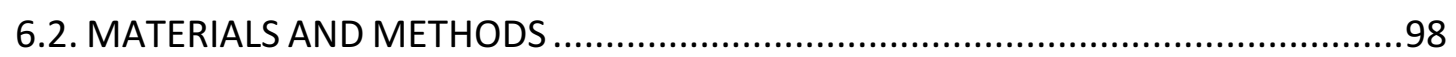

6.2.1 Animals and ethical statements ................................................................98

6.2.2. Experimental design .............................................................................98

6.2.3. Vitrified embryo transfer procedure ......................................................100

6.2.4. Growth, body weight and organ weight study .....................................101

6.2.5. Semen collection and sperm evaluation ................................................101

6.2.6. Fertility rate and number of liveborn.....................................................102

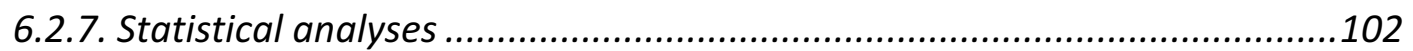

6.2.8. Transcriptomic analysis of the liver .....................................................103

6.2.9. Semi-polar and non-polar analysis of the liver metabolome.....................104

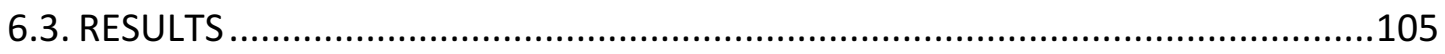

6.3.1. Establishment of the two experimental progenies (VT vS NC) across the three

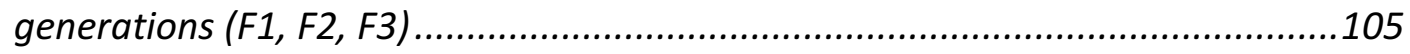

6.3.2. Growth performance, body weight and organ phenotype study ..............106

6.3.3. Sperm and fertility rate assessment ......................................................109

6.3.4. Comparative study of the liver transcriptome ..........................................109

6.3.5. Comparative study of the liver metabolome ............................................111

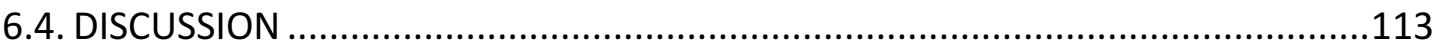

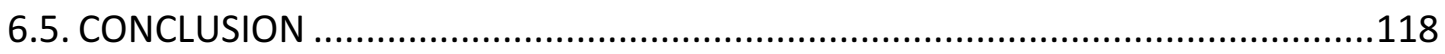

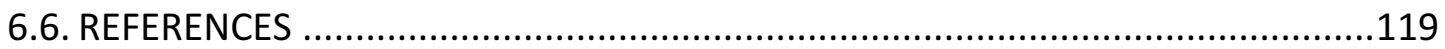

7. CHAPTER V. TRANSGENERATIONAL EFFECTS FOLLOWING VITRIFIED EMBRYO TRANSFER IN RABBITS: A MULTI-OMIC APPROACH.......................................... 129

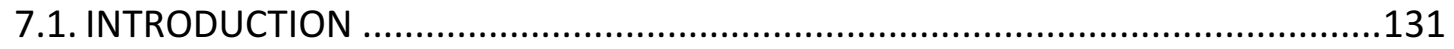

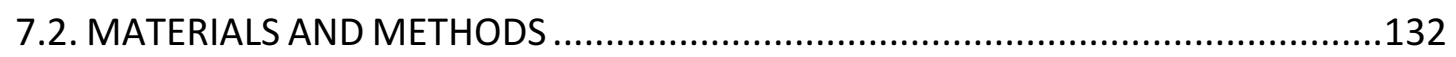

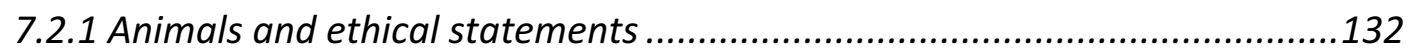

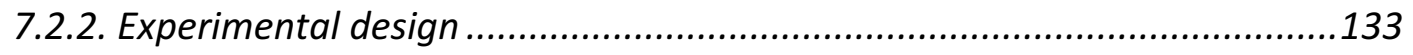

7.2.3. Determination of peripheral blood parameters .......................................134

7.2.4. Sample collection for molecular study.................................................135

7.2.5. Semi-polar and non-polar analysis of the liver metabolome......................135

7.2.6. Comparative proteomic analysis .........................................................137

7.2.7. Genome-wide DNA methylation profiling by MBD-Seq ...........................138

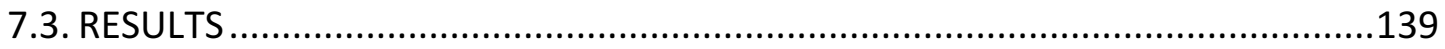

7.3.1. Peripheral blood parameters (healthy status)........................................139

7.3.2. Comparative study of the liver metabolome .............................................140

7.3.3. Comparative study of the liver proteome ................................................142

7.3.4. Comparative study of the liver epigenome...........................................144 
7.4. DISCUSSION

7.5. CONCLUSION

7.6. REFERENCES

8. GENERAL DISCUSSION

8.1. DISCUSSION AND FUTURE PROSPECTS

8.2. REFERENCES

9. CONCLUSIONS

10. ANNEX I. SUPPLEMENTARY INFORMATION - CHAPTER III. 175

11. ANNEX II. SUPPLEMENTARY INFORMATION - CHAPTER IV 183

12. ANNEX III. SUPPLEMENTARY INFORMATION - CHAPTER V 247 


\section{INDEX OF FIGURES}

Figure 1.1. In vivo vs In vitro environmental differences during the periconceptional period.

Figure 1.2. Epigenetic genome reprogramming during gamete and early embryo development.

Figure 3.1. Laparoscopic embryo transfer assisted by laparoscopy (External view)......28

Figure 3.2. Laparoscopic embryo transfer assisted by laparoscopy (Internal view).....29

Figure 3.3. Schematization of correctly loaded straw. 31

Figure 3.4. Rabbit embryos. 32

Figure 4.1. Experimental design.

Figure 4.2. Bodyweight development: comparing differences between animals naturally-conceived (NC) and those born after fresh embryo transfer (FT), vitrified embryo transfer using a ministraw (VTs), and vitrified embryo transfer using cryotop (VTc)

Figure 4.3. Growth curves: comparing differences between animals naturally-conceived (NC) and those born after fresh embryo transfer (FT), vitrified embryo transfer using a ministraw (VTs), and vitrified embryo transfer using cryotop (VTc)

Figure 4.4. Peripheral blood analysis (haematological and biochemical): comparing differences between animals naturally-conceived (NC) and those born after fresh embryo transfer (FT), vitrified embryo transfer using a ministraw (VTs), and vitrified embryo transfer using cryotop (VTc).

Figure 5.1. Experimental design. .74

Figure 5.2. Growth curves and differences in body weight between animals derived from vitrified-transferred embryos (VT) and those naturally conceived (NC). .79

Figure 5.3. Molecular analysis of liver samples obtained from adult males derived from vitrified-transferred embryos (VT) and naturally-conceived animals (NC).

Figure 6.1. Experimental design. 99 
Figure 6.2. Scatterplots showing the phenotypic raw data distributions of naturallyconceived (NC) and vitrified-transferred (VT) progenies during three generations (F1, F2, F3).

Figure 6.3. Differences in phenotypic traits between naturally-conceived (NC) and vitrified-transferred (VT) progenies during three generations (F1, F2, F3) 108

Figure 6.4. Molecular analysis of the liver samples collected from adult males derived from vitrified-transferred embryos (VT) and naturally-conceived (NC), which was compared in each generation (F1, F2, F3).

Figure 7.1. Experimental design.

Figure 7.2. Metabolite profile changes in the liver of F3 animals after vitrified embryo transfer procedure.

Figure 7.3. Protein profile changes in the liver of F3 animals after vitrified embryo transfer procedure. .143

Figure 7.4. Genome-wide methylation changes in the liver of F3 animals after vitrified embryo transfer procedure. 
Table 3.1. Efficiency of fresh rabbit embryo transfer (in vivo derived) by laparoscopy.33

Table 3.2. Viability of non-compacted vs compact vitrified morula. .33

Table 4.1. Prenatal survival. Rates of implantation, foetal losses and offspring in naturalconceived, fresh embryo transfer, vitrified embryo transfer using ministraw, and vitrified embryo transfer using cryotop.

Table 4.2. Male reproductive performance: comparing differences between naturallyconceived males and those born after fresh embryo transfer, vitrified embryo transfer using ministraw, and vitrified embryo transfer using cryotop.

Table 4.3. Female reproductive and lactation performance: comparing differences between naturally-conceived females and those born after fresh embryo transfer, vitrified embryo transfer using ministraw, and vitrified embryo transfer using cryotop.

Table 5.1. Body weight and dissection data of adult males derived from vitrifiedtransferred embryos and those naturally conceived. .80

Table 5.2. Haematological and biochemical comparison between peripheral blood of animals derived from a vitrified embryo transfer procedure (vitrified-transferred) and those naturally conceived (naturally conceived).

Table 6.1. Efficiency in the establishment of the naturally-conceived and vitrifiedtransferred progenies across three generations (F1, F2, F3). .105

Table 6.2. Ejaculates/sperm parameters and motility assessment of males from the vitrified-transferred progeny (VT) compared to those naturally conceived (NC).

Table 7.1. Haematological and biochemical comparison, assessing the transgenerational effect of the vitrified embryo transfer procedure. 140 
